\title{
The Organization of Spatial Coding in the Hippocampus: A Study of Neural Ensemble Activity
}

\author{
H. Eichenbaum, ${ }^{1}$ S. I. Wiener, ${ }^{1, a}$ M. L. Shapiro, ${ }^{2}$ and N. J. Cohen ${ }^{3}$ \\ 'Department of Biological Sciences, Wellesley College, Wellesley, Massachusetts 02181, 'Department of Psychology, \\ McGill University, Montreal, Quebec H3A-1B1, and ' ${ }^{D}$ epartment of Psychology, Johns Hopkins University, Baltimore, \\ Maryland 21218
}

\begin{abstract}
Neural activity was recorded from local groups of hippocampal single units in rats performing a spatial-memory task. The organization of functional correlates in these neural ensembles was investigated by examining the spatial relationships among the place fields of single units in each ensemble. The distance and overlap between place fields were determined together with the tuning of cellular activity to behavioral variables, including direction, speed, and turning angle during movements within place fields. The place fields of recorded neural ensembles were significantly clustered: closer in space and considerably more overlapped than chance when compared statistically with Monte Carlo simulations. Just as single units often have significant firing in more than one distinct location in the environment (subfields), the ensembles had multiple and distinct clusters of overlapping subfields. In addition, proximity and overlap between place fields were significantly, but weakly, correlated with similarity in optimal movement tuning parameters. These results suggest that the hippocampus maintains a local organization with respect to place fields despite having no apparent large-scale isomorphism with the spatial environment. The organization of multiple, clustered place fields with correlated movement tuning properties in small neural ensembles suggests the existence of functional neural ensembles serving to encode multiple sensory and behavioral aspects of a place or event. Such an organization is similar to that observed for neocortical association areas afferent to the hippocampal system.
\end{abstract}

The strong correlation of hippocampal complex-spike cell activity with specific locations occupied by a rat as it navigates through a spatial environment has provided a powerful tool for investigating hippocampal function (e.g., O'Keefe and Dostrovsky, 1971; O'Keefe and Nadel, 1978; Olton et al., 1978; McNaughton et al., 1983b; Muller and Kubie, 1987; O'Keefe and Speakman, 1987). These studies have shown that the activity of complex spike cells in rats exploring the environment

\footnotetext{
Received Aug. 18, 1988; revised Jan. 20, 1989; accepted Jan. 20, 1989.

We acknowledge the generous support of NIH grant NS18744, BRSG grants to Wellesley College, NSF grants BNS8721157 and BNS 8810095, and a grant from the Sloan Foundation to N.J.C. We also thank Carol Ann Paul for technical assistance, and Apostolos Georgopoulos and Nestor Schmajuk for many helpful discussions.

Correspondence should be addressed to Howard Eichenbaum, Science Center, Wellesley College, Wellesley, MA 02181.

a Present address: Laboratoire de Physiologie Neurosensorielle, 15, ru de l'Ecole de Médecine, 75270 Paris Cedex 06 France.

Copyright @ 1989 Society for Neuroscience $0270-6474 / 89 / 082764-12 \$ 02.00 / 0$
}

is characterized by "place fields," i.e., areas of the environment in which these units are reliably activated. However, some studies have also demonstrated that the animal's spatial position does not fully determine firing rate. As shown in the preceding paper (Wiener et al., 1989), the rat's movement direction, speed, and turning angle all influence the firing rate of complex spike cells in a spatial-navigation task. Furthermore, complex spike cells are activated during the performance of nonspatial tasks, such as discrimination learning with visual (Wible et al., 1986), olfactory (Eichenbaum et al., 1987; Wiener et al., 1989), or auditory cues (Foster et al., 1986) in rats, as well as during classical conditioning in rabbits (Berger et al., 1983) and recognition of specific words and faces in humans (Heit et al., 1988).

While hippocampal unit activity can be reliably related both to behavioral events and to spatial location, spatial parameters provide a more easily identifiable set of continuous, measurable dimensions that might be systematically mapped onto the anatomy of the hippocampus. Thus, we investigated the functional organization of the hippocampus by examining the spatial relationships among place fields and between place fields and firing correlates of movement (second-order spatial) parameters in ensembles of CA1 neurons. The activity of neighboring hippocampal complex spike cells was recorded simultaneously as rats performed a spatial navigation task. For each individual neuron within the ensemble of cells recorded, the place field was identified, its spatial relationship to other place fields within the ensemble was quantified, and the activity of the cell in relation to movement variables was determined. In this way it was possible to evaluate the spatial extent of the environment included by all place fields in each ensemble, the degree of organization of place fields within the ensemble, and the correlation between the distance between place fields and the similarity of movement correlates within the ensemble.

Single CAI neurons have place fields that can include relatively large areas of an environment, and nearby units can have place fields distributed throughout that environment (O'Kcefe, 1979). Furthermore, no systematic relationship has yet been observed between places in the environment and anatomical locations in the hippocampus (O'Keefe, 1979; Barnes et al., 1983). Thus, in terms of global topography, no orderly relationship has been observed between the region of hippocampus in which a particular neuron is located and the region of space occupied by its place field.

Place fields recorded from neighboring hippocampal cells could conceivably be organized in many ways, but 2 contrasting views of brain organization offer reasonable models. The hippocampus might be organized as a massively parallel distributed network, with the representation of any given place in the environment 
distributed across the network of neural elements (e.g., McNaughton and Morris, 1987; Rolls, 1987). In accordance with this model, single neurons would be expected to be sensitive to a significant portion of and, possibly, multiple places within the environment; and there would not be any obligatory topography characterizing the place fields of nearby cells. This model of organization is consistent with O'Keefe and Speakman's (1987) finding that even a small group of neighboring hippocampal neurons could represent most of the environment. Alternatively, the hippocampus might be organized in the manner of the primary sensory cortices, in which neighboring cells have partially overlapping receptive fields and are similar on other functional measures, and slightly more distant neurons have receptive fields spaced proportionately further apart and are more different on other functional measures (Mountcastle, 1957). In accordance with this model, neighboring cells within a hippocampal ensemblc should have ovcrlapping placc ficlds in a restricted part of the environment and other functional commonalities, much as neurons within a column of visual cortex are sensitive to the same small portion of visual field and prefer the same orientation of stimuli (Hubel and Wiesel, 1977).

\section{Materials and Methods}

Descriptions of electrode construction, surgery, behavioral apparatus, and testing, and determination of place fields and movement tuning are provided in detail in the preceding paper (Wiener et al., 1989). Accordingly, only a brief description of each will be offered here, accompanied by a detailed description of special analyses for characterizing the distribution of place fields in simultaneously recorded cells.

\section{Electrodes and surgery}

The multichannel microelectrodes used in this study were composed of ten $25 \mu \mathrm{m}$ Teflon-coated nichrome wires bundled into a 26 gauge cannula and attached to a vertically driveable connector (Eichenbaum et al., 1977; Kubie, 1984). Just before implantation, the exposed wires were cut to equal length and then teased apart so that recordings could be taken from cells horizontally displaced over approximately a $1-\mathrm{mm}$ diameter area. Rats were tranquilized with acepromazine, given atropine to prevent excessive salivation, and anesthetized with pentobarbital. Body temperature was maintained with a $39^{\circ} \mathrm{C}$ thermal pad. The head was placed in a stereotaxic frame with bregma and lambda level. The electrode was implanted at coordinates relative to bregma: posterior 3.2 $\mathrm{mm}$, lateral $1.8 \mathrm{~mm}$, and $1-1.5 \mathrm{~mm}$ below the cortical surface. The electrode base was secured with dental cement and stainless steel skull screws. One screw over the frontal bone scrved as the electrical ground.

\section{Behavioral apparatus and training}

Unit activity was recorded while rats performed a spatial-navigation task similar in memory demands to Olton's radial-arm maze paradigm (Olton and Samuelson, 1976). The apparatus was a $0.4 \mathrm{~m}^{2}$ arena with metal walls slanted at $15^{\circ}$ to prevent the rat from bumping the head stage. A $2 \mathrm{~cm}^{2}$ plastic water cup was mounted in each corner of the arena. A cul-de-sac on one wall, used as an odor port in the previous paper (Wiener et al., 1989), offered an orienting spatial stimulus here. Position and movements of the rat were monitored by locating an incandescent light bulb mounted on the rat's headstage using a TV tracking system.

On each trial, the rat was required to move to the centcr of the arcna, activating a pulsating tone that indicated the availability of reward upon approach to any of 4 water cups. The rat was required to return to the center, again initiating the tone, prior to approaching successive water cups. An appropriately initiated approach to each cup was rewarded only once; repeated visits to water cups within a trial or visits not initiated by first moving to the arena center were not rewarded. A 30 sec intertrial interval was imposed by preventing trial initiation after all cups were visited. Normal rats acquired this task rapidly and made few errors even in sessions with many trials. Each recording session included $24-96$ trials.

\section{Unit recording and discrimination}

Prior to each session, the electrode was driven up to $80 \mu \mathrm{m}$ and allowed to settle for at least $3 \mathrm{hr}$. Unit activity was recorded and isolated using a multichannel amplifier (300-1000 $\mathrm{Hz}$ filter cutoffs) and window discriminator array. Often more than one unit was discriminated from the same electrode wire simultaneously by directing the recorded analog signal to multiple spike windows set with nonoverlapping thresholds The accuracy of discrimination at each spike window was monitored throughout the recording session by matching an audio signal from the discriminator output to the visual display of the analog waveform. To eliminate recording artifacts, signals from a separate unit channel with no detectable unit activity were passed to a "wide-open" window (low threshold just above background noise and no high threshold), and al spike events within $100 \mathrm{msec}$ of the noise were ignored in post hoc analyses. Despite these measures, there remains a significant potentia for incomplete unit isolation or inclusion of recording artifacts on multiple channels simultaneously; either type of error might result in an apparent coincidence of place fields or movement-related activity across channels. Therefore, several post hoc control analyses were performed to test if the observed organizational properties appeared only as a consequence of these potential errors (see below).

\section{Analyses}

Analyses proceeded in 2 stages: The first stage determined the boundaries of place fields and tuning to movement variables for each cell separately. The methods for this analysis are described in detail in the preceding paper (Wiener et al., 1989); a brief description is included here. The second stage determined the relationships among place fields and between place fields and various movement variables within the group of simultaneously recorded cells, hereafter called the ensemble.

Stage 1. Determination of place fields and movement tuning. The boundaries of place fields were characterized by measuring areas of the arena reliably associated with increased firing upon repeated visits. The arena was divided into an array of $18 \times 16$ (the larger dimension included the cul-de-sac) $2.5 \mathrm{~cm}^{2}$ "pixels." To increase the reliability of mcasurcment, a firing rate was detcrmined for cach "visit" to a pixcl, defined as at least 3 sequential $50 \mathrm{msec}$ observations within a $3 \times 3$ pixel area centered by the target pixel. A mean firing rate for each pixel and the grand mean and SE were calculated for all pixels. The place field was defined as the set of pixels at least $2.33 \mathrm{SE}$ above the grand mean and having at least one border shared with at least 2 other pixels meeting the statistical criterion. Many place fields were composed of 2 or more distinct groups of pixels meeting these criteria; these are called subfields.

Movement tuning properties were determined by measurements on the series of observations of the rat's location within each place field, or within each subfield separately if the place field had multiple subfields. The instantaneous speed of movement associated with each location was estimated using the distance traveled from $100 \mathrm{msec}$ ( 2 observation points) beforc to $100 \mathrm{msec}$ after that location. The instantaneous direction of movement associated with each location was estimated using the sum of vectors computed from location observations over the same $200 \mathrm{msec}$ period used for speed determinations. The instantaneous turning angle at each location was estimated as the arc subtended by vectors drawn between that location point and points $100 \mathrm{msec}$ before and after that point. For each variable, measurements were sorted into 8 bins that covered the range of measures for that variable. The reliability of tuning to each variable was evaluated by comparing firing rates across the 8 bins with a $\chi^{2}$ analysis. Further analyses were performed for each variable only in cases with significant tuning $(p<0.05)$.

Stage 2. Determination of relationships among place fields. The spatial distribution of place fields within cell ensembles was analyzed by measuring the average distance between nearest neighbors among subfields and the amount of overlap of all the ficlds in the ensemble. The center of each subfield was defined as the pixel closest, on average, to all other pixels in the subfield; the distance to the nearest neighbor was determined by comparing distances between all pairs of subfield centers. The average nearest-neighbor distance among the subfields was used as an overall measure of place field separation.

The overlap of place fields was measured in terms of the fraction of the environment actually covered by the ensemble of place fields compared with the amount of the environment that could have been covered given the size of the place fields. This measure, referred to as the overlap ratio, eliminated the effect of place-field size and was computed as the 
number of unique pixels that were included in any place field divided by the total of the sizes of individual place fields. For example, if a pixel were part of 2 place fields, it would be counted once (as a unique pixel) in the numerator and twice in the denominator. Hence, a low ratio indicated a high degree of overlap and a ratio of 1.0 indicated no overlap. The lower bound of this ratio was limited by the number of subfields. However, all statistics compared the measures on ensembles of actually recorded place fields with those of simulated place fields matched for size and number of subfields. Statistical analyses of average nearestneighbor distance and overlap ratio were based on Monte Carlo simulations designed for each ensemble. Each Monte Carlo simulation sample used a set of place fields identical in size and form to that of the place fields in the recorded ensemble, but each of the simulated place fields was positioned and oriented randomly within the environment; the orientations and distances among the subfields within each place field were maintained. For each of the 62 recorded ensembles, nearestneighbor distance and overlap ratio were calculated for each of 1000 Monte Carlo samples, and the percentile rank of the measure from the recorded ensemble was determined from the distribution of the Monte Carlo simulation.

Further analyses assessed the extent to which place field distance and overlap tended to correlate with the differences or similarity of the movement correlates associated with cellular activity as the rat passed through the subfields. Two measures relating each pair of subfields from different cells within an ensemble were taken. First, the center of each subfield was determined (as described above) and the center-to-center distance between each pair was measured. Second, the number of pixels shared by each pair of fields was counted and normalized to a percent overlap as a function of the smaller of the 2 fields; thus, $0 \%$ indicated no overlap and $100 \%$ indicated that the smaller subfield was entirely enclosed within the larger one. Comparisons between pairs of subfields on movement variables were based on calculations of optimal tuning for each parameter: Optimal speed was taken as the movement speed associated with the maximal firing rate. Optimal direction of movement and turning angle were calculated as the vector sum of firing rates across directions and turning angles, respectively. The difference on each movement parameter was calculated as the absolute difference between optimal tuning measures for each pair of subfields, with the exception of subfields of the same cell. To determine the relationship between place field clustering and differences in movement correlates, distance and overlap were separately correlated with differences in the optimal movement speed, direction, and turning angle.

\section{Results}

In all, 62 ensembles of 3-11 (mean $=5.2$ ) cells were analyzed. As reported in the preceding paper, $75 \%$ of these cells had place fields varying in size from 1 to $29 \%$ of the area of the arena. The majority of cells were also tuned to movement variables: $69 \%$ for speed, $77 \%$ for direction, and $62 \%$ for turning angle. Other details on the spatial and other firing properties of these cells are reported in the accompanying paper (Wiener et al., 1989).

\section{Relationships between place fields in neural ensembles}

Place fields of neighboring hippocampal neurons were significantly clustered in space: they were closer to one another and overlapped more than would be expected by chance, and they were often grouped into multiple clusters of overlapping subfields. Of the 62 ensembles recorded, 47 (77\%) had smaller nearest-neighbor distances and 48 had smaller overlap ratios among place fields than the mean of their matched Monte Carlo simulations. The remaining ensembles were further apart and overlapped less than their Monte Carlo simulations (Fig. 1). Sign tests showed that these distributions differ from that expected by chance (for distance: $z=4.06, p<0.001$; for overlap: $z=4.32, p<0.001)$. Moreover, as shown in Figure 1, the distribution of percentile rankings for both measures on ensembles was skewed so that the modal value was within the 5 th percentile for both measures. Examples of place field clusters are shown together with statistical analyses of distance and overlap in Figures 2 and 3. The case shown in Figure $2 A$ is an example of a small ensemble in which all 3 place fields observed included the center-right of the arena. In this case, the average nearestneighbor distance for the ensemble was in the fourth percentile of the Monte Carlo distribution, indicating closer proximity than one would expect by chance. The overlap ratio for the recorded group was smaller than that of any of the Monte Carlo runs, indicating much greater overlap than expected by chance. Similar results were obtained with larger ensembles. In the case of the large ensemble shown in Figure $2 B$, the measures of place field distribution also indicate greater clustering than expected by chance. The average nearest-neighbor proximity was in the 9th percentile of the Monte Carlo distribution, and the overlap ratio was in the 1 st percentile of the Monte Carlo distribution.

Often multiple, distinct clusters of place fields occurred. In the case shown in Figure $3 A$, a cluster of many subfields is seen near the center of the arena and another, involving only 2 subfields, at one of the reward cups. In the case shown in Figure $3 B$, there were several clusters involving groups of $2-4$ subfields each. Note that each of the 4 cells recorded had multiple subfields that appeared within various subsets of the clusters. The average nearest-neighbor and overlap ratio measures for both cases indicate that these distributions are highly unlikely by chance. Because of the presence of multiple clusters, the proportion of the arena covered by all of the place fields combined was as high as $75 \%$ with a mean of $33 \%$.

Although the tendency of recorded ensembles to exhibit abovechance clustering of place ficlds was confirmed statistically with extensive Monte Carlo testing, there are 2 possible sources of confounding of the data that need to be addressed. One is the possible effect on the clustering measures of a bias in the placefield locations toward the edges of the environment. The other is the possibility that poor unit isolation in extracellular recordings causes neural potentials from the same cells to be recorded in separate channels. These 2 possibilities will be discussed in turn.

\section{Possible effects of place-field location bias}

The Monte Carlo analyses upon which the statistical tests of place field clustering were based chose place-field locations in a purely random fashion, favoring no particular portion of the arena. However, the actual location of place fields recorded from cells in ensembles were biased away from the center and towards the edges of the arena (see preceding paper, Fig. 5). This biasing of place-field locations in neurons actually recorded compared with those used in the Monte Carlo simulations is potentially important because it could contribute to the increased clustering of place field locations seen in the recorded ensembles. That is, even if the place fields in any recorded ensemble were located entirely independently of one another, the location bias might by itself cause an increase in clustering over that seen in the Monte Carlo simulations. In order to evaluate the possible contribution of this bias to the observed clustering, the same clustering analyses were performed on the place fields after recombining them artificially to assure their independence. The place fields obtained from the 62 recorded ensembles were placed in a pool from which 62 neural groups were constructed, each consisting of place fields from 3-11 cells, matching in number and size the set of recorded ensembles. The place fields of these constructed groups had the very same location bias as in the recorded ensembles, but the cells comprising each constructed 


\section{A. AVERAGE NEAREST-NEIGHBOR}

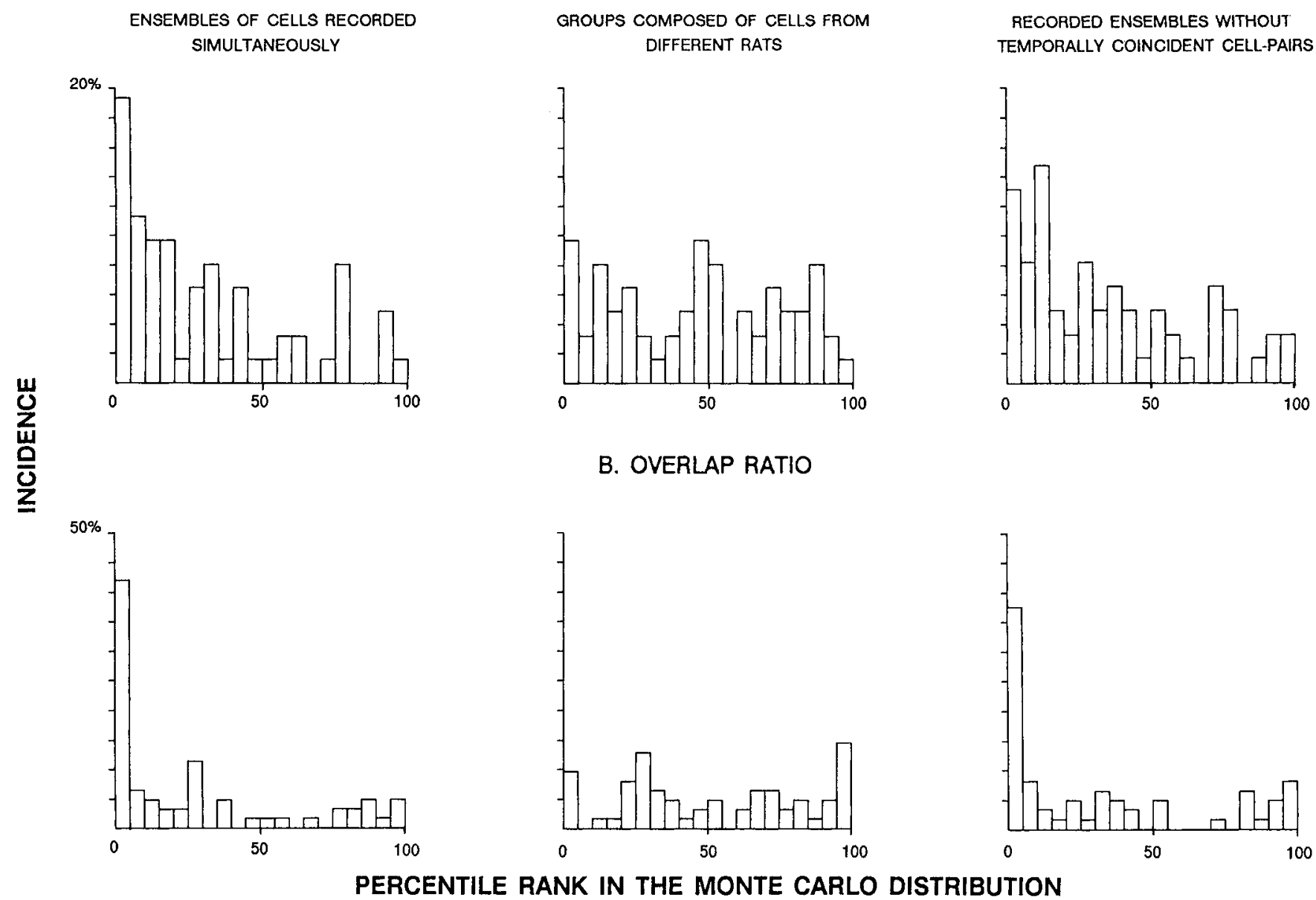

Figure 1. Nearest-neighbor and overlap ratio measures of neural ensembles compared with Monte Carlo distributions. The distribution of recorded neural ensembles, of groups composed of cells from different rats, and of recorded ensembles with temporally coincident cell pairs eliminated are shown as a percentile ranking of their Monte Carlo distributions. $A$, The distribution of average nearest neighbors of recorded ensembles is highly skewed, with most subfields closer than would be expected by chance (left graph). The skewed distribution of place-field distances was not altered significantly when pairs of cells firing in high temporal coincidence were eliminated (right graph). In contrast, the distribution of groups of independent cells, composed of cells taken randomly from different rats, is relatively flat (middle graph). $B$. The distribution of overlap ratios of recorded ensembles is also highly skewed, with most subfields more overlapped than would be expected by chance (left graph). The overlap ratio distribution was not altered significantly by eliminating pairs of cells firing in high temporal coincidence (right graph). Independent groups of cells had a flat distribution (middle graph).

group were taken from different animals to assure their functional independence.

Place fields of these "constructed" groups showed little preference in their tendency to cluster, with a range of values from the same small distance and large overlap as that observed in recorded ensembles, to a much greater distance than cxpected by chance and almost no overlap (Fig. 1). Thirty-four of 62 $(55 \%)$ groups had smaller average nearest neighbors than the mean of their Monte Carlo runs $(z=0.76, p=0.47)$ and 31 $(50 \%)$ had smaller overlap ratios $(z=0, p=0.5)$, a distribution no different than would be expected by chance. A direct comparison between the results based on data from the recorded ensembles versus the groups constructed from cells from different rats showed that the number of sessions above and below the mean of the Monte Carlo distribution differed significantly both for the average nearest-neighbor $\left(\chi^{2}=5.13, p<0.02\right)$ and for the overlap ratio $\left(\chi^{2}=8.93, p<0.01\right)$ measures, indicating that the place fields of recorded ensembles were more clustered than the groups constructed of independently recorded cells.
While particular cases of groups of cells taken from different rats had place fields clustered to the same degree as those of the recorded ensembles, many others had no more clustering, or less clustering, than that expected by chance. An example case of each of the latter will be shown here. Examples of analyses on 2 groups composcd of cells selected randomly from different rats are given in Figure 4. The case shown in Figure $4 A$ had an average nearest neighbor and an overlap ratio very near the mean for each respective measure in the Monte Carlo distributions. Groups of this type may appear to be clustered by visual inspection, but statistically are no more clustered than expected by chance, according to our measures. The case shown in Figure $4 B$ had an average nearest neighbor in the 93rd percentile of the Monte Carlo distribution and an overlap ratio greater than the largest of the Monte Carlo simulations. Groups of this type appear have almost no overlap, an organization that is unlikely by chance.

The foregoing analysis demonstrates that the bias of placefield location in independently selected cells does not by itself 
A

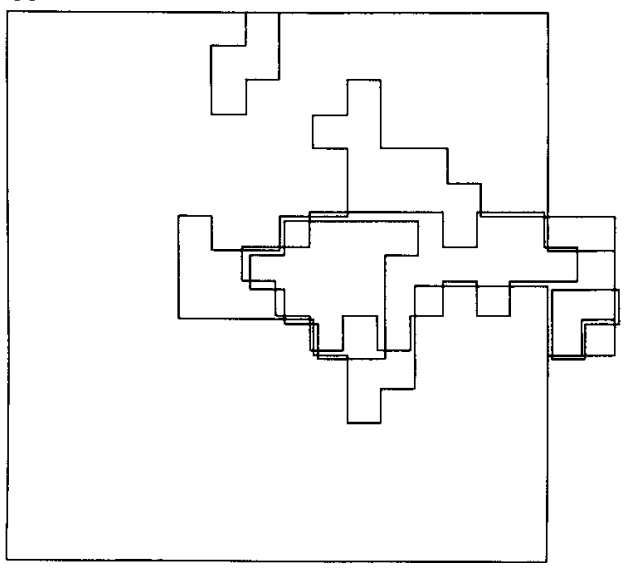

B

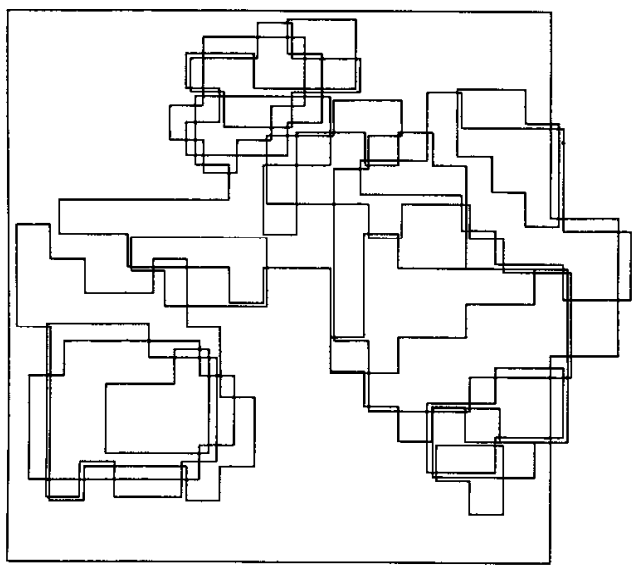

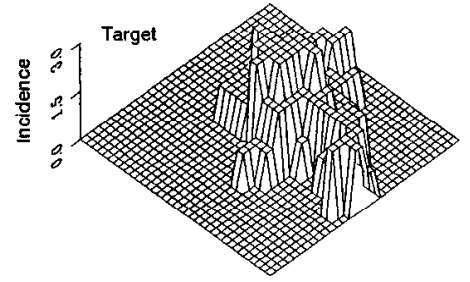
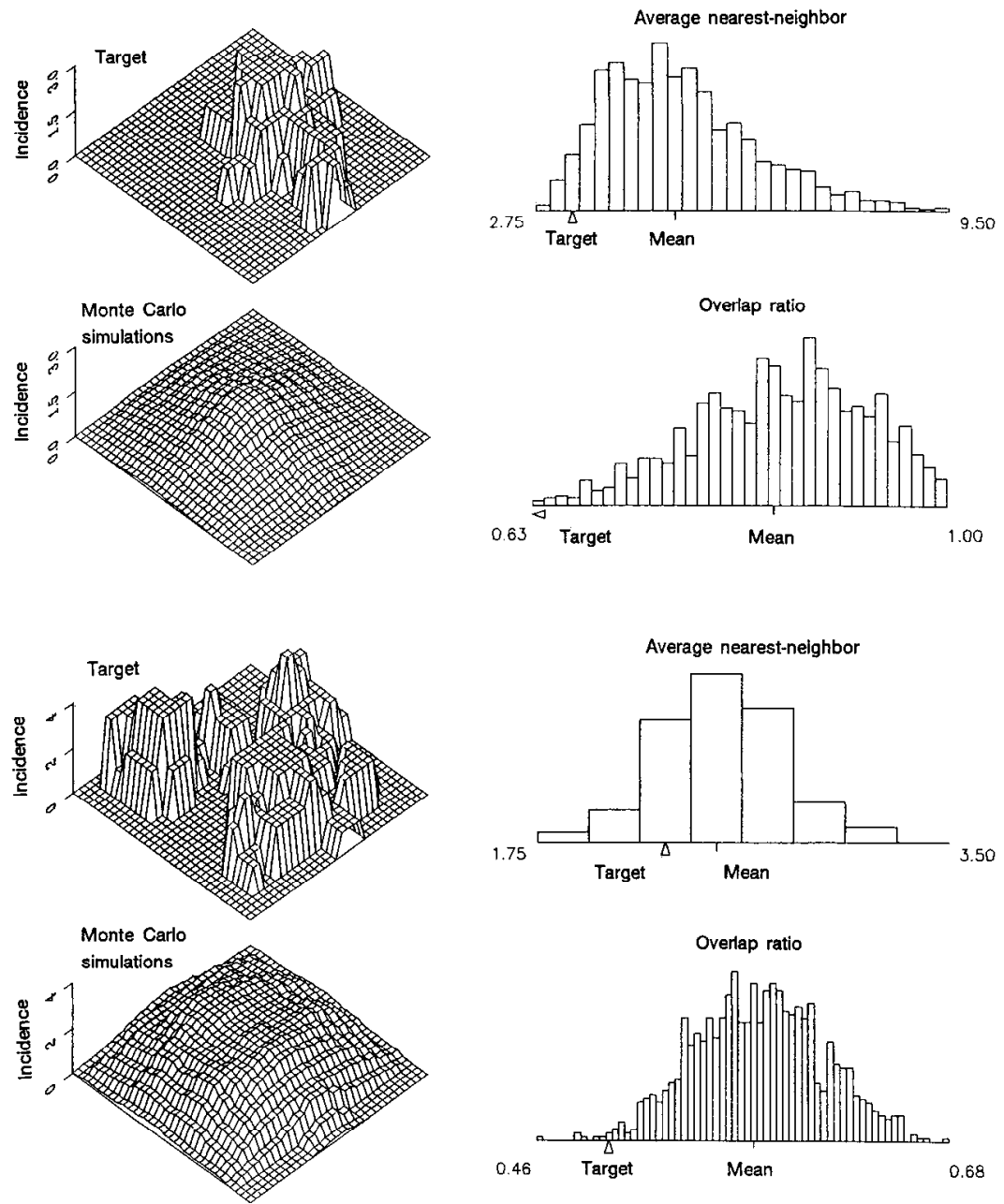

Figure 2. Analyses of ensemble activity. $A$ and $B$ are cases of small and large ensembles, respectively. Left, An outline of the experimental chamber is shown from a top view of the environment; the location of the cul-de-sac is indicated on the right side. The boundaries of the place field of each cell is outlined with overlapping edges of place fields shifted slightly to aid their visualization. Center. The 3-dimensional graphs indicate the spatial coincidence of pixels, i.e., the number of times a given pixel was included in a place field, both for the target recorded ensemble (above) and the average spatial coincidence for the 1000 Monte Carlo runs (below). Right, The distributions of average nearest-neighbor and overlap ratio for the Monte Carlo runs are shown, with the arrowhead indicating the value of each measure for the target recorded ensemble. The range of the Monte Carlo distribution was determined by the size and number of the place fields used in the simulation: larger and more numerous ensembles were more constrained by the size of the apparatus than were smaller ensembles. The range of each Monte Carlo distribution is noted numerically and illustrated by the width of the bars in the figure. $A$, In this example of a small recorded ensemble, 3 cells had 5 subfields that included the centerright of the chamber, distributed over 54 pixels, or $20 \%$ of the chamber. The average nearest neighbor in the neural group was 3.45 pixels. This measure was in the fourth percentile of the Monte Carlo distribution, indicating closer proximity than one would expect by chance. The overlap ratio for the recorded group was 0.59, smaller than that of any of the Monte Carlo runs (range $=0.63-0.92$ ), indicating much greater overlap than expected by chance. $B$, Similar results were obtained with larger ensembles. Here, 9 cells had a total of 16 subfields distributed over 138 pixels, or $52 \%$ of the chamber. Again, the measures of placc-ficld distribution indicate greater clustering than expected by chance. The average nearestneighbor proximity was 2.28 pixels, which was in the 9 th percentile of the Monte Carlo distribution. The overlap ratio was 0.49 , which was in the 1st percentile of the Monte Carlo distribution.

cause significant clustering and therefore cannot provide an explanation of the clustering observed in the ensembles of neighboring cells.

\section{Possibility of poor unit isolation}

Another possible confounding of the clustering observed in recorded ensembles may be due to recording neural potentials from the same cells in closely spaced wires (McNaughton et al., 1983a) or to poor discrimination of cells recorded from the same wire. That is, in any multiple-cell recording study based on extracellular recording methods, it is impossible to make un- ambiguous identifications of individual neural potentials with distinct neurons. Without the use of intracellular recording methods, which are not available for use in chronic studies of behaving animals, it is possible that potentials recorded from different channels may reflect a single cell. This would, of course, lead to an inappropriate conclusion of common place fields and increase the estimate of clustering.

To evaluate the extent to which this was a factor in the reported data, the likelihood of coincidence (within $1 \mathrm{msec}$ ) among all pairs of cells recorded simultaneously was measured. Recording from the same single cell on 2 channels should be re- 

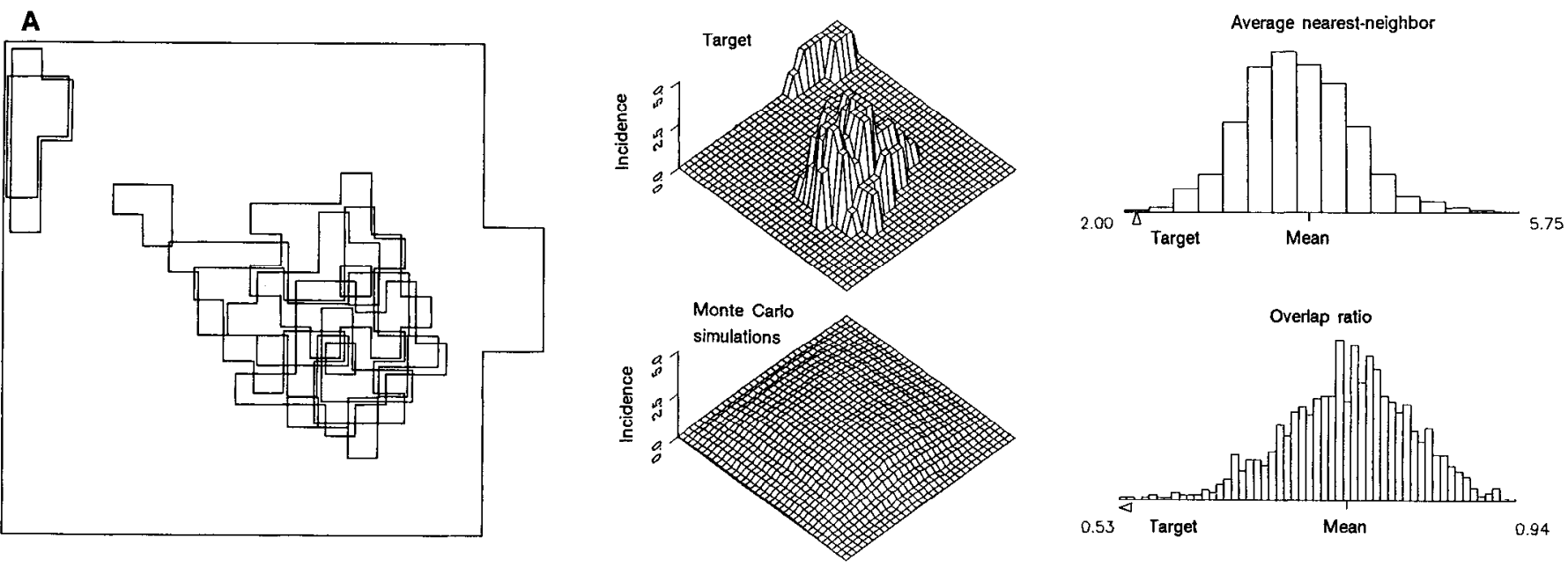

B
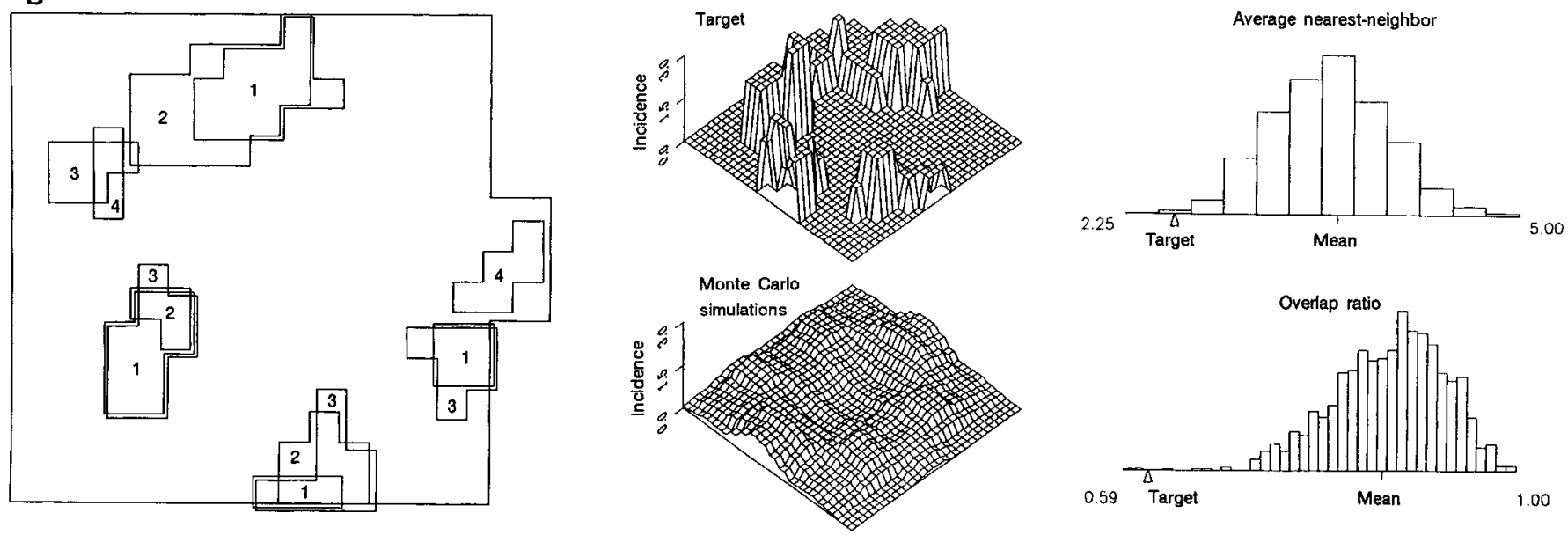

Figure 3. Distinct and multiple place fields were clustered in recorded ensembles. $A$, An ensemble of 7 cells had 9 subfields that clustered in 2 distinct areas of the maze. $B$, An ensemble of 4 cells had 13 subfields that clustered in 4 distinct areas of the maze. The subfields of each cell are shown by numbers $1-4$. Place fields $1-3$ had subfields in each of the 4 overlapping subfield clusters. Conversely, each of these place fields had shifted and overlapping subfields in 4 distinct areas of the chamber.

flected in a near-perfect temporal coincidence of spike events on both channels. Lower firing coincidence could reflect either a combination of poor cellular isolation on both channels or the recording of potentials from 2 separate cells that tend to coactivate. Since significant coactivation has been observed among hippocampal neurons recorded by electrode sites separated by at least $100 \mu \mathrm{m}$ (Kuperstein et al., 1986), both possible explanations of high temporal coincidence must be considered realistic. Furthermore, one might expect the likelihood for coactivation among cells to be great when the demand for hippocampal function is high, such as in the spatial-navigation task employed in the present experiment. To evaluate the effects of high temporal coincidence on the observed findings, cases in which temporal coincidences of greater than $50 \%$ were noted and Monte Carlo analyses were performed again excluding the data of one cell chosen randomly from high-coincidence cell pairs.

The temporal coincidence was over $50 \%$ in 20 out of 745 ccllpairs evaluated. Figure 5 shows the place fields of ensembles excluding 1 (Fig. $5 A$ ) and 2 (Fig. $5 B$ ) cells that were members of high temporal-coincidence pairs; in both cases, the remaining place fields still had much greater clustering than expected by chance. The distribution of percentile rankings in the Monte Carlo analyses of the recorded ensembles excluding one member of each high temporal-coincidence pair is very similar to what was reported above for all cells (Fig. 1), indicating that the effect of temporal coincidence on measures of clustering was minimal. Using the overlap measure: 43 out of 61 ensembles had greater overlap than expected by chance $(z=3.2, p=0.0007)$.

Two other related tests were performed to further examine the possibility of poor unit isolation. First, the same control analysis as that described above was repeated, but with a stricter criterion: all cell pairs with $20 \%$ or greater coactivation were rejected. Under this strict criterion, several ensembles were lost from the analysis because the number of cells fell below the minimum of 3 , but the distribution in the remaining ensembles still exhibited morc overlap than expected by chance ( 34 out of 52 groups; $z=2.2, p=0.015$ ). Second, even though the previous 
A
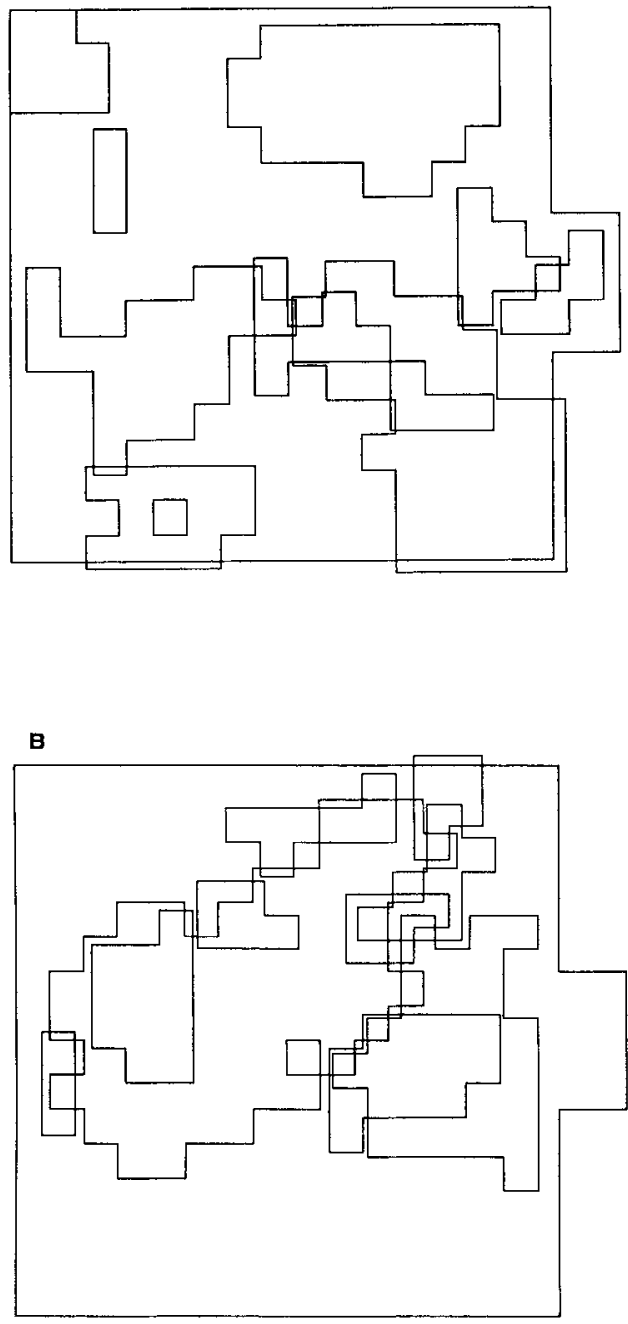
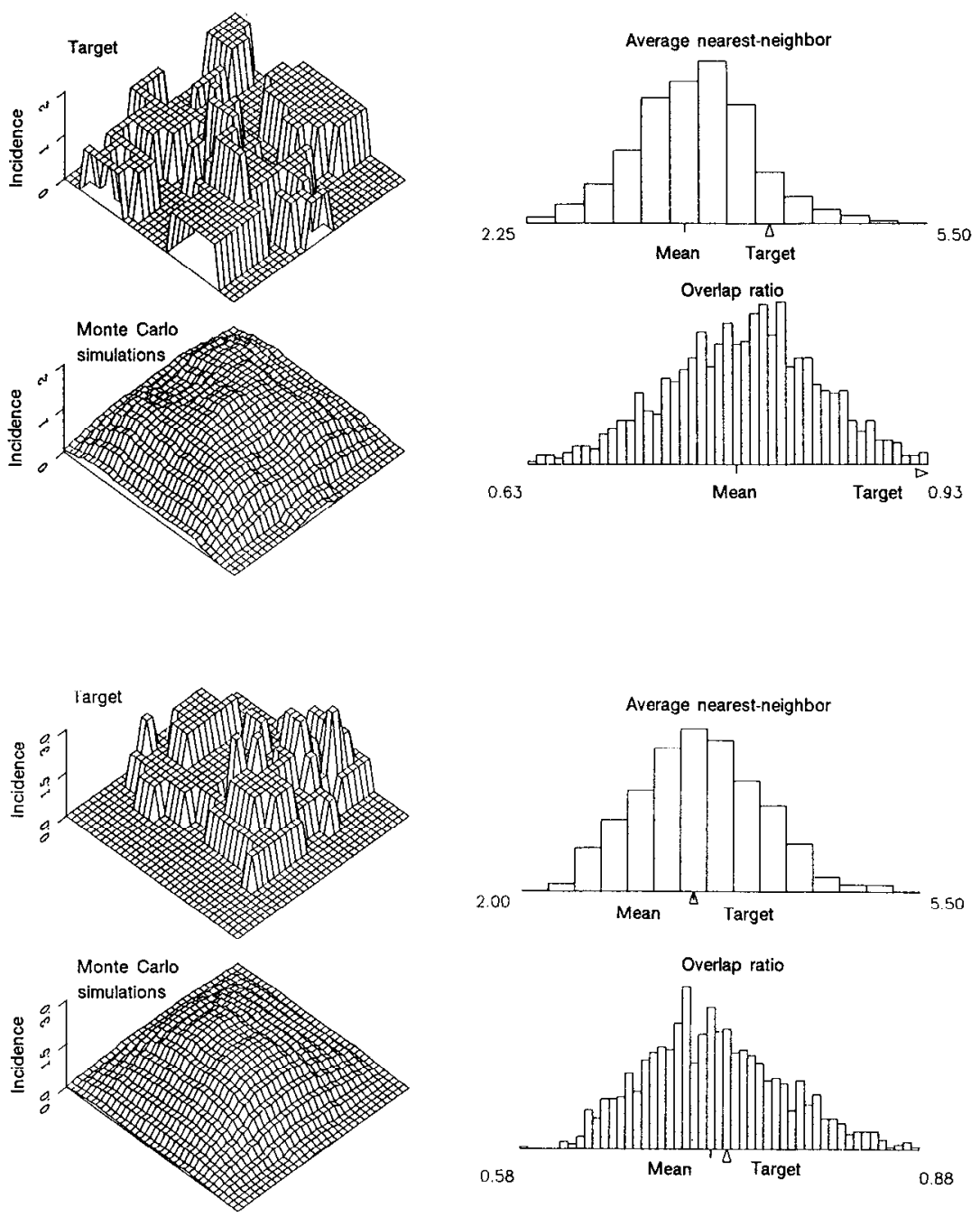

Figure 4. Examples of groups of cells selected randomly from different rats. A, Five independent cells had 9 subfields covering 136 pixels (51\%) of the chamber. This group illustrates a distributed, almost nonoverlapping group of place fields, with a significantly higher overlap-ratio and nearest-neighbor measures than expected by chance, falling into the 93rd and 94th percentiles of the Monte Carlo distribution. $B$, Five independent cells had 10 subfields that covered 118 pixels $(45 \%)$ of the chamber. The fields appeared clustered, but in fact the measures of clustering fell in the middle of the Monte Carlo distribution of overlap ratio, and thus this degree of clustering was likely to occur by chance. Similarly, the nearestneighbor analysis showed the subfields were, on average, at the distances expected by chance.

analyses should have eliminated the specific effect of poor discrimination of cells recorded on the same wire, the same control test was repeated, but in this test, only one unit from each wire was included. Although this control also eliminated a substantial number of ensembles with less than 3 remaining cells from the analysis, the exclusion of multiple channels recorded on each wire had little effect on the distribution of place fields in remaining ensembles. Sixty-seven percent of the ensembles $(28$ out of 42) showed greater overlap than would be expected by chance $(z=2.16, p<0.015)$.

\section{Correlation of clustering and similarity in movement tuning}

Differences in the optima of movement tuning variables (speed, direction, and turning angle) between pairs of subfields of cells recorded simultaneously were directly correlated with distance between the subfields and inversely correlated with the overlap between the subfields (Table 1). The direction of the correlations, as reflected in the slopes of the regressions, indicate that the closer and more overlapping place subfields tended to have similar optimal speed, directional, and angular tuning. The correlation coefficients were low, indicating that differences in speed, direction, and turning angle each contributed only modestly to the variability in place field proximity and overlap. Nonetheless, each correlation (with the exception of overlap $\times$ speed-difference) was highly significant.

It is notable that the low correlations indicate that cells with overlapping place fields do not have identical movement tuning properties, as would be expected if the described overlap was due to recording the same cell on multiple electrode channels. Thus, the outcome of these correlations provides further evidence for rejecting the hypothesis that the observed clustering is an artifact of poor unit isolation. 

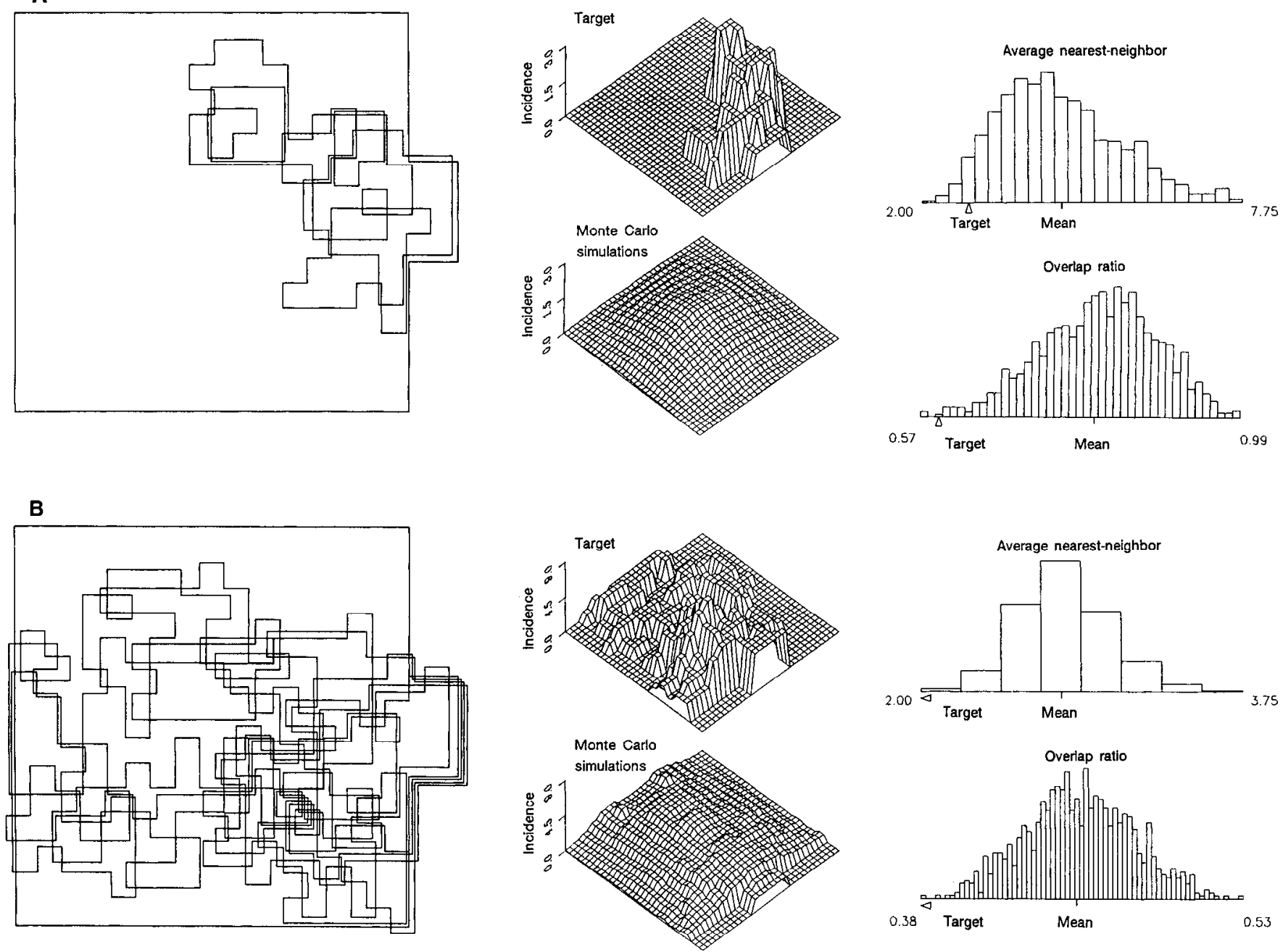

Figure 5. Examples of recorded ensembles with temporally coincident cell pairs eliminated. If a pair of cells had greater than 50\% temporal coincidence, one of the cells was eliminated from further statistical analysis. $A$, A small ensemble with 4 cells had 7 subfields covering 58 pixels (22\%) of the chamber. Despite the elimination of one coincident cell, this recorded ensemble had significantly clustered place fields. The nearestneighbor measure was in the 4th percentile, and the overlap ratio was within the 1 st percentile of their respective Monte Carlo distributions. $B$, A large ensemble with 9 cells had 15 subfields covering 157 pixels $(59 \%)$ of the chamber. Elimination of 2 coincident cells did not alter the significant clustering of the place fields. The nearest-neighbor and overlap ratio measures were both outside the range of their respective Monte Carlo distributions.

\section{Discussion}

\section{Properties of ensembles of hippocampal CA1 neurons}

The activity of local ensembles of cells revealed 4 basic properties of the functional organization of place fields in the hippocampus. Local ensembles of hippocampal CA1 units (1) have a set of place fields that cover a considerable portion of the total environment explored by the animals; (2) have place fields that are more clustered than would be expected by chance; (3) have distinct, multiple clusters of place fields with partial overlap in each cluster; and (4) demonstrate a slight but significant direct correlation between clustering and similarity in associated movement correlates. Each of these properties will be discussed in turn.

Small ensembles of cells have a set of place fields that cover a substantial portion of the total environment explored by the animals. This finding is consistent with previous work showing that the place fields of a group of 8 neighboring cells covered nearly the entire environment of a radial maze (O'Keefe and Speakman, 1987). Here, as much as $75 \%$ of the total area of the arena was included by the place fields of as few as 11 neighboring cells within an ensemble.

Place fields recorded in ensembles tend to cluster. Analyses of place-field proximity and overlap showed that neighboring cells were likely to have nearby and overlapping place fields, clustering to a greater extent than was observed either in random arrangements of comparably sized and shaped fields produced in Monte Carlo simulations or in arrangements of place fields for groups constructed from the data of neurons recorded in different animals compared with their Monte Carlo simulations. Importantly, the observed clustering occurred in the absence of any anatomically or physiologically demonstrated "patches" of hippocampus; rather, it occurred in fortuitously selected groups of neighboring cells within the sampled region of CAl. The 
Table 1. Correlation of distance and overlap between place fields or subfields with differences in movement speed ( 706 pairs), direction (1106 pairs), and turning angle (679 pairs) of place cells

\begin{tabular}{ll} 
Slope & $\begin{array}{l}\text { Correlation } \\
\text { coefficient }\end{array}$ \\
\hline
\end{tabular}

Distance vs difference in

$\begin{array}{llll}\text { Speed } & +2.13 & 0.12 & <0.001 \\ \text { Direction } & +0.11 & 0.14 & <0.0001 \\ \text { Angle } & +0.10 & 0.11 & <0.004 \\ \text { Overlap vs difference in } & & \\ \text { Speed } & -0.99 & 0.07 & 0.081 \\ \text { Direction } & -0.11 & 0.19 & <0.0001 \\ \text { Angle } & -0.09 & 0.12 & <0.0013\end{array}$

Correlations are reported as absolute values; $p$ gives the significance level of the correlation.

present finding of clustering differs from O'Keefe's conclusion that neighboring units have nonoverlapping place fields ( $\mathrm{O}^{\prime} \mathrm{Keefe}$, 1979; O'Keefe and Speakman, 1987). O'Keefe (1979) had observed that "Neighboring cells ... a are as likely to have fields widely separated in an environment as they are to have ones which are contiguous." That qualitative description, if taken literally, characterizes the present data quite well: Within any ensemble, fields were observed in one of several clusters. Thus, pairs of fields were almost equally likely to be in different clusters and therefore to include different regions of space as to be in the same cluster and therefore to include overlapping regions of the environment. Such clustering is, as we have seen, significantly beyond that expected by random arrangement of the individual place fields.

In more recent studies, O'Keefe and Speakman (1987) reported that "units recorded from the same or neighboring locations in the hippocampus had different fields in the same environment." The conflict between this conclusion and the present findings may be due in part to methodological differences between the studies. Whereas the present work employed a bundle of $25 \mu \mathrm{m}$ wire electrodes sampling an area with a 1 mm radius, O'Keefe and Speakman (1987) used a stereotrode with a pair of $25 \mu \mathrm{m}$ wires separated by $6 \mu \mathrm{m}$. Accordingly, it is possible that the dispersed place fields reported in the stereotrode work came from adjacent or nearly adjacent cells, and the clustered place fields found in the present work with microwire bundles came from somewhat more distant neighbors. The demonstration that nonadjacent neighbors would have a systematic clustering not seen in neurons that are more closely adjacent would be revealing. However, O'Keefe and Speakman's (1987) own work suggests otherwise: First, 17 of the 44 recorded ensembles showed significant spatial correlation between the location of pairs of place fields. Fourteen were correlated positively, indicating clustered fields, and 3 were correlated negatively, indicating dispersed fields. Second, the distance between neurons was estimated and closer units had higher spatial correlations than more distant units. Rather than being due to methodological differences, any discrepancy between O'Keefe and Speakman's (1987) conclusions and those offered here seems more likely to be one of interpretation. Indeed, inspection of O'Keefe and Speakman's (1987) group of 8 neighboring neurons recorded simultaneously (their figure 12) appears to show the very clustering that was actually quantified statistically in the present report. Taken together, the findings strongly suggest a local order in hippocampus in which ensembles of neighboring cells fire when the rat is in a particular location or locations.

Place fields of neighboring cells within ensembles are characterized by multiple clusters of subfields with partial overlap within each cluster. Just as single CS units often have place fields composed of distinct subfields (e.g., Wiener et al., 1989; O'Keefe and Speakman, 1987, figures 7, 11, 13; Muller et al., 1987, figures 7,8 ), ensembles of CS units were found here to have multiple clusters of overlapping place fields or subfields (Fig. $3 B$ ). Interpreted in light of the present findings, the one large ensemble shown by O'Keefe and Speakman (1987, figure 11) was composed largely of cells with multiple subfields clustered either on the $+90^{\circ}$ and $-90^{\circ}$ maze arms or the $0^{\circ}$ and $180^{\circ}$ arms, foreshadowing the organization of multiple clusters revealed in greater detail here. The fact that the subfields cluster across neurons within an ensemble provides suggestive evidence for the physiological significance of subfields; cells in any given ensemble may respond redundantly in association with the rat's presence in several separate regions of the environment.

A dissimilarity can be noted between the organization of multiple distinct clusters found among the place fields studied here and the organization of partial shifted overlap found in the receptive fields of cortical sensory neurons. The pattern of partial overlap observed in sensory cortices is manifested by an orderly shifting of receptive fields corresponding to the systematic sampling of adjacent neurons in the relevant cortical map (e.g., Mountcastle, 1957; Hubel and Wiesel, 1977). It is possible, but unlikely, that partial overlap in hippocampal ensembles resembles what is seen in cortical sensory maps-i.e., that there is a systematic relationship between the relative position of the hippocampal neuron within an ensemble and the relative position of its place fields with respect to the others. The current technique did not permit this possibility to be assessed definitively. However, the partially shifted overlap of receptive fields in sensory cortices occurs within a large-scale topographic mapping of a receptor surface; no similar global topographic mapping of an environment has been found within the hippocampus (Barnes et al., 1983). Moreover, even though the present mcthods were not the most direct for the analysis of global organization, the observed organization of multiple distinct clusters of place fields covering a large fraction of the environment is inconsistent with the possibility of a large-scale isomorphic topography of Euclidean space in the hippocampus.

Differences in movement tuning optima were directly correlated with distance and inversely correlated with overlap of place fields among cells in the ensembles. Comparisons of pairs of subfields within ensembles showed that cells with increasingly proximal or overlapping place fields tended towards similarity in their preferences for particular movement speeds, directions, and turning angles and, conversely, cells that had fields that were more distant or separated were less likely to have similar movement correlates. Such an organization in hippocampus would be similar to the way in which neighboring cells within a column of striate cortex represent the same portion of the visual field and have similar orientation tuning. Note, however, that the correlation coefficients were low. This may have been due to the coarseness of the various measures of proximity, overlap, and movement, or to the existence of many other factors that might influence cell firing, not controlled or measured here. In addition, the model of linear regression is not entirely appropriate to revealing "nested" organizations or columns. Even in primary visual cortex, one would predict a direct cor- 
relation between receptive field clustering and orientation tuning only over a small set of columns (a hypercolumn); at distances beyond that no correlation would be expected. Despite these limitations, the correlations of proximity and overlap with similarity in optimal movement parameters revealed a significant relationship, consistent with the possibility that the hippocampus is functionally organized into neuronal ensembles representing "hyperfields," analogous to hypercolumns in striate cortex, with movement parameters nested within spatial positions.

\section{The value of representing space with multiple clusters of place} fields

Having observed clustering and partial overlap of place fields within ensembles of neighboring hippocampal neurons, it behooves us to ask about the spatial processing function that such local organization may serve. Two functions of the observed local organization for spatial representation come to mind. The first is increased spatial resolution, assuming for the moment that the purpose of place fields is to represent one's spatial location. If instead of coding spatial position in terms of the area represented by a place field, spatial position was coded by the area represented by a "functional field," composed of the intersection of several place fields, there would be a significant reduction in the amount of uncertainty about the animal's location in space signaled by hippocampal unit activity. The functional fields created by partial overlap are smaller than the contributing place fields and, with the amount of overlap actually observed here, provides a greater degree of spatial resolution than would be the case if the place fields were entirely nonoverlapping. This would exemplify the coarse coding scheme described by neural network theorists (Hinton et al., 1986).

The second function relates to the nesting of movement correlates in "functional fields" or hyperfields. That is, considering neural ensembles as a functional unit, movement speed, direction, and turning angle can be represented along with spatial position in the same neurons. Note that movement correlates may also, in principle, serve to further reduce positional uncertainty by providing information about a particular view (direction of movement), looming (movement speed), and sweep (the change in direction of movement). Furthermore, the putative nested organization of hyperfields provides a new way to view the interactions of spatial and other behavioral correlates in hippocampal unit activity (e.g., Wible et al., 1986; Eichenbaum et al., 1987; Wiener et al., 1989). According to this view, nonspatial information is represented along with spatial information in the hyperfield, and it is predicted that most or all other behavioral correlates of hippocampal CS activity, including olfactory and other mnemonic correlates, should be observed to nest within space [if indeed space is the primary correlate (see below)].

\section{Towards an understanding of the functional organization of the hippocampus}

What do these findings imply about the functional organization of the hippocampus? Clearly, the hippocampus resembles neither the orderly topographic maps of primary sensory cortices nor the random distribution of features across the entire set of neural elements expected on certain views of massively parallel neuronal networks (Rumelhart and Zipser, 1986). Despite the absence of any larger-scale topographic organization, a local order emerges: Substantial overlap exists among clusters of place fields of local neuronal groups and clustered place fields tend to have similar movement correlates. This pattern of organization observed among neurons is consistent with each of several different representational schemes that are characteristic of primate neocortical association areas afferent to the hippocampal formation (see Van Hoesen, 1982): (1) a nonisomorphic map of space, like that seen in visual area MT; (2) a representation of spatial position relative to body orientation, like that seen in the posterior parietal and prefrontal cortices; or (3) a representational scheme in which space is not the fundamental variable being mapped but is a component of, or coincides at multiple loci with the underlying functional correlate, like the representation of objects in inferotemporal cortex. Each of these provides a possible model for viewing hippocampal representation; they will be considered in turn.

1. A nonisomorphic map. One possible account of hippocampal representation consistent with the present findings is that the hippocampus is composed of many physiologically distinct "patches" corresponding to different regions of space, each patch containing neurons with similar place fields and other tuning characteristics. Interestingly, some of the ensembles recorded here had place fields that were considerably less clustered and more distant than expected by chance. These cases, if significant, are consistent with the idea that patches may exist, and the electrode bundle straddled more than one of them.

Another possible nonisomorphic mapping, consistent with the present findings, involves a continuous nonisomorphic map rather than one composed of discrete bounded entities. A "patchy" physiology might emerge stochastically from an input gradient that causes nearby cells to have a higher probability of receiving similar inputs than more distant cells. Neighboring cells would tend to share more of their input than would more distantly separated cells, and might therefore be expected to have place fields in similar portions of the environment, thus exhibiting the clustering seen in the present results. This organizational scheme would be analogous to that for the representation of visual fields in area MT of primates. The organization of this tertiary visual area has been described as a nonisomorphic, but continuous gradient that maps visual receptive fields with multiple representations of the same spatial loci, anisotriopies, under- and overemphases, and a tendency of neighboring cells to have similar speed and directional correlates (Maunsell and Van Essen, 1983, 1987). The view of the hippocampus as having a continuous nonisomorphic map of this type can be distinguished empirically from the possibility that the hippocampus is composed of patches by performing systematic recordings across adjacent areas of the hippocampus. If the hippocampus were made up of distinct patches, moving across any given area of hippocampus with electrodes would be expected to reveal a discontinuous collection of place fields clustered in some regions of space and then another collection of place fields clustered in some other regions of space, and so on. That is, it should be possible to discern discontinuities in the place fields, corresponding to the boundaries between patches. On the gradient view, however, moving across any given area of hippocampus with electrodes would be expected to reveal a continuous, if not orderly function of distance between hippocampal neurons and distance between sets of corresponding place field clusters.

2. Representation of spatial position relative to body orientation. Another view of hippocampal representation follows from the proposal offered in the preceding paper that unit activity reflects the animal's position and movements in space relative 
to significant landmarks (Wiener et al., 1989). Many cells fired primarily as the animal approached the corners of the arena to obtain rewards or returned to the center to initiate another approach. The most complete description of the functional correlate of these neurons involved a specification of both movement and position. This interpretation was further supported by the occasional observation of multiple correlates of the same cell (or neighboring cells) that had spatially symmetrical place fields and movement correlates (see Wiener et al., 1989, Fig. $6 D$ ). These data suggest that an individual cell or neighboring cells can encode multiple spatially directed movements each relative to locations with particular similarities defined by the environmental characteristics (in Fig. $6 D$, opposite corners). Such an encoding of places and movements relative to body orientation would be similar to the representation of visual fields in parietal cortex (area 7a) in primates. In that neocortical association area, cells have receptive fields that are retinotopic, but the magnitude of visual responses are gated by ocular anglc, resulting in a representation of places in space relative to the orientation of the head (Andersen et al., 1985). It is notable that the receptive fields of parietal cortex are not topographic, nor is topographic representation required to model the visual response properties of these cells (Zipser and Andersen, 1988).

An alternative model for hippocampal organization that also involves representation of relative position and movements is provided by the dorsomedial frontal cortex. In that premotor area, cells fire during the execution of either eye or arm movements towards targets at particular locations in space, relative to body position (Mann et al., 1988; see also Niki and Watanabe, 1976; Funahashi et al., 1988). The representations of movements in space in these areas are analogous to the active-movement and relative place-field correlates that were identified in hippocampal neurons in the preceding paper (Wiener et al., 1989).

3. Space is not the primary dimension encoded. So far, discussion has been limited to the possible spatial mapping role of hippocampal neurons. Yet, as has already been made clear, CA1 units in the hippocampus also fire in relation to other taskrelevant variables. The present investigation raises several questions about the other aspects of processing thought to be carried out by the hippocampus: Do other correlates of hippocampal activity reveal functional overlap in neural ensembles? Does some nonspatial dimension better follow the model of sensory cortex, revealing local partially shifted overlap and large-scale topographic mapping in the hippocampus? Does "clustering" of responses in local ensembles persist across different tasks and environments? These questions, each critical to understanding the functional architecture of the hippocampus, remain to be tested.

As we search for the appropriate analog of neocortical organization with which to compare all the findings on the hippocampus, we should consider yet one more association area-the inferotemporal cortex (IT), one of the highest visual processing areas of primates, which sends both indirect and direct (Yukie and Iwai, 1988) inputs to the hippocampus. The trigger feature and receptive field properties of IT have been studied extensively (e.g., Gross et al., 1972; Perret et al., 1982; Desimone et al., 1984). IT neurons have receptive fields of varying size, many with very large or bilateral fields. These visual neurons are maximally responsive to 2 - and 3-dimensional patterns, and many are highly selective for specific familiar objects, including faces. Furthermore, while the representation of neither their receptive fields nor their trigger features is topographically organized, "clusters" of neighboring cells have similar receptive field and trigger feature properties (Desimone and Gross, 1979; Gochin et al., 1988). Thus, the receptive field organization described for IT is similar to that observed here for place fields in the hippocampus. Perhaps we should consider that the place correlate is more apparent than real, and that hippocampal cells, like IT neurons, may represent "places" as independent complex "objects" more than as contiguous elements within a larger map of space. This view would go far to explain the lack of largescale organization of place fields in the hippocampus. Furthermore, this perspective would bring closer together disparate descriptions of spatial and nonspatial variables encoded by hippocampal neurons, consistent with our view that places, defined by the spatial relations of multiple stimuli, are just one example of relational processing in the hippocampus (Eichenbaum and Cohen, 1988).

\section{References}

Andersen, R. A., G. K. Essick, and R. M. Siegel (1985) Encoding of spatial location by posterior parietal neurons. Science 230:456-458.

Barnes, C. A., B. L. McNaughton, and J. O'Keefe (1983) Loss of place specificity in hippocampal complex spike cells of senescent rat. Neurobiol. Aging 4: 113-119.

Baylis, G. C., E. T. Rolls, and C. M. Leonard (1985) Selectivity between faces in the responses of a population of neurons in the cortex of the superior temporal sulcus of the monkey. Brain Research 342 . 91-102.

Berger, T. W., P. C. Rinaldi, D. J. Weisz, and R. F. Thompson (1983) Single-unit analysis of different hippocampal cell types during classical conditioning of the nictitating membrane response. J. Neurophysiol. 50: $1197-1219$.

Desimone, R., and C. G. Gross (1979) Visual areas in the temporal area of the macaque. Brain Res. 178: 363-380.

Desimone, R., T. D. Albright, C. G. Gross, and C. Bruce (1984) Stimulus-selective properties of inferotemporal neurons in the macaque. J. Neurosci. 4: 2051-2062.

Eichenbaum, H., and N. J. Cohen (1988) Representation in the hippocampus: What do hippocampal neurons encode? Trends Neurosci. 11: 244-248.

Eichenbaum, H., D. Pettijohn, A. M. Deluca, and S. L. Chorover (1977) Compact miniature microelectrode-telemetry system. Physiol. Behav. 18: 1175-1178.

Eichenbaum, H., M. Kuperstein, A. Fagan, and J. Nagode (1987) Cuesampling and goal-approach correlates of hippocampal unit activity in rats performing an odor discrimination task. J. Neurosci. 7: 716732.

Foster, T. C., E. P. Christian, R. E. Hampson, K. A. Campbell, and S. A. Deadwyler (1986) Sequential dependencies regulate sensory evoked responses of single units in the rat hippocampus. Brain Res. 408: 86-96.

Funahashi, S., C. J. Bruce, and P. S. Goldman-Rakic (1988) Memory fields: Directional tuning of delay activity in the dorsolateral prefrontal cortex of the rhesus monkey. Soc. Neurosci. Abstr. 14: 868.

Gochin, P. M., E. K. Miller, C. G. Gross, and G. L. Gerstein (1988) Circuit properties of inferior temporal cortex in the macaque. Soc. Neurosci. Abstr. 14: 11.

Gross, C. G., D. B. Bender, and C. E. Roche-Miranda (1972) Visual properties of inferotemporal neurons of the macaque. J. Neurophysiol. 35 : 96-111.

Heit, G., M. E. Smith, and E. Halgren (1988) Neural encoding of individual words and faces by the human hippocampus and amygdala. Nature 333: 773-775.

Hinton, G. E., J. L. McClelland, and D. E. Rumelhart (1986) Distributed representations. In Parallel Distributed Processing: Explorations in the Microstructure of Cognition, Vol. 1, pp. 77-109, MIT Press, Cambridge, MA

Hubel, D. H., and T. N. Wiesel (1977) Ferrier Lecture. Functional architecture of macaque monkey visual cortex. Proc. R. Soc. London [Biol.] 198: 1-59.

Kubie, J. L. (1984) A driveable bundle of microwires for collecting 
single-unit data from freely moving rats. Physiol. Behav. 32: 115118.

Kuperstein, M., H. Eichenbaum, and T. VanDeMark (1986) Neural group properties in the rat hippocampus during the theta rhythm. Exp. Brain Res. 61: 438-442.

Mann, S. E, T. Thau, and P. H. Schiller (1988) Conditional taskrelated responses in monkey dorsomedial frontal cortex. Exp. Brain Res. 69: 460-468.

Maunsell, J. H. R., and D. C. Van Essen (1983) Functional properties of neurons in the middle temporal visual area of the macaque monkey. I. Selectivity for stimulus direction, speed, and orientation. J. Neurophysiol. 49: 1127-1147.

Maunsell, J. H. R., and D. C. Van Essen (1987) Topographic organization of the middle temporal visual area in the macaque monkey: Representational biases and the relationship to callosal connections and myeloarchitechtonic boundaries. J. Comp. Neurol. 266: 535555.

McNaughton, B. L., and R. G. M. Morris (1987) Hippocampal synaptic enhancement and information storage within a distributed memory system. Trends Neurosci. 10: 408-415.

McNaughton, B. L., J. O'Keefe, and C. A. Barnes (1983a) The stereotrode: A new technique for simultaneous isolation of several single units in the central nervous system from multiple unit records. J. Neurosci. Methods 8: 391-397.

McNaughton, B. L., C. A. Barnes, and J. O'Keefe (1983b) The contributions of position, direction, and velocity to single cell unit activity in the hippocampus of freely moving rats. Exp. Brain Res. 52: 4149.

Mountcastle, V. B. (1957) Modality and topographic properties of single neurons of cat's somatic sensory cortex. J. Neurophysiol. 20: $408-434$

Muller, R. U., and J. L. Kubie (1987) The effects of changes in the environment on the spatial firing of hippocampal complex-spike cells. J. Neurosci. 7: 1951-1968.

Muller, R. U., J. L. Kubie, and J. B. Ranck (1987) Spatial firing patterns of hippocampal complex-spike cells in a fixed environment J. Neurosci. 7: 1935-1950.

Niki, H., and M. Watanabe (1976) Prefrontal unit activity and delayed response: Relation to cue location versus direction of response. Brain Res. 105: 79-88.
O'Keefe, J. A. (1979) A review of hippocampal place cells. Prog. Neurobiol. 13: 419-439.

O'Keefe, J., and J. Dostrovsky (1971) The hippocampus as a spatial map. Preliminary evidence from unit activity in the freely-moving rat. Brain Res. 34: 171-175.

O'Keefe, J., and L. Nadel (1978) The Hippocampus as a Cognitive Map, Oxford U. P., Oxford, UK.

O'Keefe, J. A., and A. Speakman (1987) Single unit activity in the hippocampus during a spatial memory task. Exp. Brain Res. 68: 127.

Olton, D. S., and R. J. Samuelson (1976) Remembrance of places passed: Spatial memory in rats. J. Exp. Psychol.: Anim. Behav. Proc. 2: $97-116$.

Olton, D., M. Branch, and P. J. Best (1978) Spatial correlates of hippocampal unit activity. Exp. Neurol. 58: 387-409.

Perrett, D. I., E. T. Rolls, and W. Caan (1982) Visual neurons responsive to faces in the monkey temporal cortex. Exp. Brain Res. 47: 329-342.

Rolls, E. T. (1987) Information representation, processing, and storage in the brain: Analysis at the single neuron level. In Neural and Molecular Mechanisms of Learning, Springer-Verlag, Berlin.

Rumelhart, D. E., and D. Zipser (1986) Feature discovery by competitive learning. In Parallel Distributed Processing: Explorations in the Microstructure of Cognition, Vol. 1, pp. 151-193, MIT Press, Cambridge, MA.

Van Hoesen, G. W. (1982) The parahippocampal gyrus: New observations regarding its cortical connections in the monkey. Trends Neurosci. 5: 345-350.

Wible, C. G., R. L. Findling, M. Shapiro, E. J. Lang, S. Crane, and D. S. Olton (1986) Mnemonic correlates of unit activity in the hippocampus. Brain Res. 399: 97-110.

Wiener, S. I., C. A. Paul, and H. Eichenbaum (1989) Spatial and behavioral correlates of hippocampal neuronal activity. J. Neurosci. 9: 2737-2763.

Yukie, M., and E. Iwai (1988) Direct projections from the ventral TE area of the inferotemporal cortex to the hippocampal field CA 1 in the monkey. Neurosci. Lett. $88: 6-10$.

Zipser, D., and R. A. Andersen (1988) A back-propagation programmed network that simulates response properties of a subset of posterior parietal neurons. Nature 331: 679-684. 